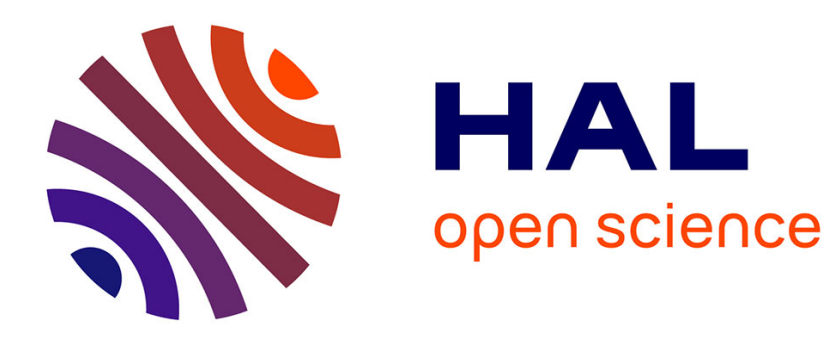

\title{
Spéculation et marchés dérivés du pétrole
}

Julien Chevallier

\section{To cite this version:}

Julien Chevallier. Spéculation et marchés dérivés du pétrole: Eléments d'analyse économique et statistique. 2010. halshs-00458916

\section{HAL Id: halshs-00458916 \\ https://shs.hal.science/halshs-00458916}

Preprint submitted on 22 Feb 2010

HAL is a multi-disciplinary open access archive for the deposit and dissemination of scientific research documents, whether they are published or not. The documents may come from teaching and research institutions in France or abroad, or from public or private research centers.
L'archive ouverte pluridisciplinaire HAL, est destinée au dépôt et à la diffusion de documents scientifiques de niveau recherche, publiés ou non, émanant des établissements d'enseignement et de recherche français ou étrangers, des laboratoires publics ou privés. 


\title{
Spéculation et marchés dérivés du pétrole : Éléments d'analyse économique et statistique
}

\author{
Julien Chevallier ${ }^{1}$ \\ Université Paris Dauphine
}

\begin{abstract}
Résumé :
Cet article fournit des éléments d'analyse économique et statistique concernant les marchés dérivés du pétrole, et notamment le «pic » de prix enregistré en juillet-août 2008. Les principales explications de la littérature sont recensées, tout en mettant l'accent sur les évolutions en cours. Au total, cet article met en évidence une nouvelle articulation entre les fondamentaux «physiques » du marché du pétrole qui ont prévalu jusqu'au début des années 2000, et l'émergence de nouveaux fondamentaux "financiers » suite aux réformes des marchés dérivés aux Etats-Unis en 2003 et la montée en puissance des agents "non commerciaux» sur ce marché. Enfin, des pistes d'exploitation de cette expérience récente sont suggérées sous la forme de recommandations de politiques publiques de régulation des marchés dérivés du pétrole.
\end{abstract}

Mots-clefs : Pétrole ; Spéculation ; CFTC ; Marchés Dérivés

\footnotetext{
${ }^{1}$ Julien Chevallier est Membre du Centre de Géopolitique de l'Energie et des Matières Premières (CGEMP) et du Laboratoire d'Economie de Dauphine (LEDa). Il est également Chercheur Associé avec le laboratoire EconomiX-CNRS et le Grantham Institute for Climate Change à Imperial College London. Adresse pour correspondance: Place du Maréchal de Lattre de Tassigny, 75775 Paris Cedex 16, France. Email : julien.chevallier@dauphine.fr Les opinions exprimées dans ce document n'engagent que l'auteur, et non les institutions auxquelles il est affilié. L'auteur reste seul responsable des éventuelles erreurs ou omissions.
}

Remerciements: L'auteur souhaite remercier Jean-Marie Chevallier, Michel Laffitte ainsi que les membres du Groupe de Travail sur la Volatilité du Prix du Pétrole - Frédéric Baule, Frédéric Lasserre, Ivan Odonnat, Edouard Viellefond dont le rapport a été remis à Mme. Christine Lagarde, Ministre de l'Economie, de l'Industrie et de l'Emploi le 09 février 2010. L'auteur remercie également tous les experts consultés à cette occasion à la CFTC, US Treasury, US Department of State, Federal Reserve, EIA, Congressional Research Service, US Senate, US Department of Energy, CSIS, PFC Energy, Banque Mondiale, FMI, Deutsche Bank (Washington, DC USA) et à la Commission Européenne: DG MARKT, DG ECFIN, DG TREN (Bruxelles). 


\section{Introduction}

L'augmentation de la volatilité des prix du pétrole, particulièrement depuis 2008, soulève de nombreuses questions quant à la détermination du prix du pétrole et au jeu complexe des interdépendances entre les marchés physiques et les marchés financiers, et entre leurs fondamentaux respectifs. L'intention de cet article est de livrer ici une revue de littérature aussi exhaustive que possible de la volatilité des produits dérivés du pétrole. Les travaux de recherche sur le sujet ont été très nombreux au cours des dernières années et les controverses académiques qu'ils suscitent sont parfois toujours en cours. La réalisation de ces études se heurte le plus souvent à une très grande opacité concernant aussi bien les données physiques que les données financières. Le champ des corrélations qui peuvent être faites à partir des prix des produits pétroliers est infini et les travaux aboutissent parfois à des résultats contradictoires.

Notre intention, dans cet article, est de mettre en évidence quelques développements récents concernant l'évolution des prix des produits dérivés du pétrole. Nous proposons d'analyser le fonctionnement récent des marchés pétroliers pour savoir si la volatilité des prix résulte ou non d'une transformation structurelle de ces derniers qui accordent une place grandissante à la sphère financière. Ceci nécessite de se pencher sur l'évolution des marchés pétroliers sur les dix dernières années, avant de pouvoir apprécier les risques d'une volatilité persistante dans le futur. En plus des traditionnels fondamentaux «physiques », nos résultats mettent en évidence l'émergence de nouveaux fondamentaux « financiers » liés à l'émergence d'acteurs «non-commerciaux » sur les marchés dérivés du pétrole, en lien avec les réformes institutionnelles mises en place aux Etats-Unis dans les années 2000. Enfin, nous proposons quelques mesures de politiques publiques afin de limiter les effets potentiellement négatifs de la volatilité sur l'économie à la lumière de ces expériences récentes.

La suite de l'article est composée sous la forme suivante. La Section 1 décrit quelques caractéristiques statistiques des produits dérivés du pétrole. La Section 2 rappelle les principaux fondamentaux sur ces marchés. Enfin, la Section 3 apporte des éléments d'explication de la crise de juillet-août 2008, ainsi que des suggestions de politique publique. 


\section{Un marché volatil...}

La série du prix du pétrole (New York Mercantile Exchange - NYMEX - en US\$/baril) est caractérisée par une très grande volatilité, comme l'indique la figure 1 ci-dessous :

\section{Figure 1 : Prix du pétrole NYMEX en base quotidienne du 24 juin 1988 au 12 juin} 2009

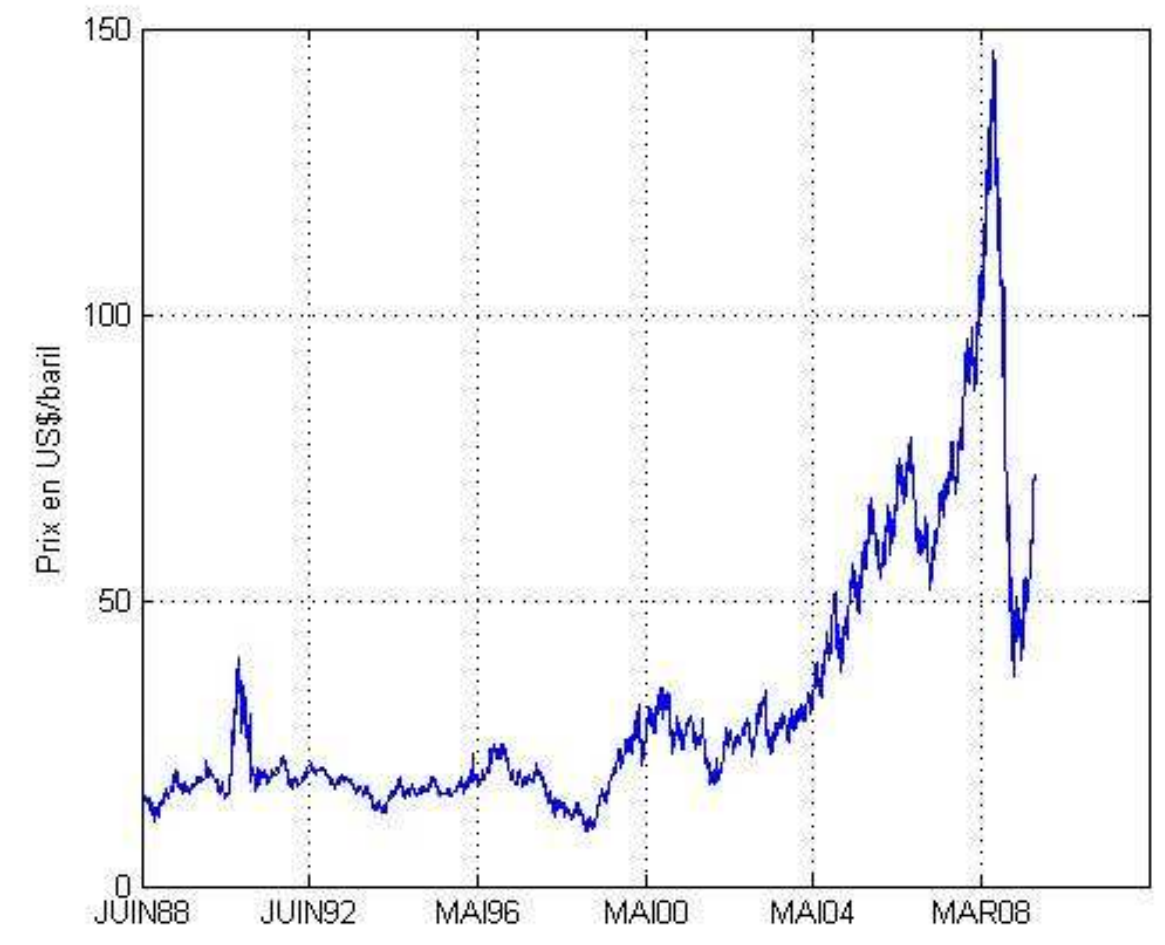

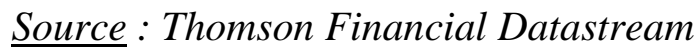

La rupture de tendance identifiée entre les mois de mars et d'août 2008 fait l'objet d'une attention toute particulière, car celle-ci serait due non aux fondamentaux bien connus du marché du pétrole (détaillés ci-après), mais à un pur phénomène de spéculation financière. Examinons plus précisément les propriétés statistiques de la série de prix du pétrole NYMEX dans le Tableau 1: 
Tableau 1 : Statistiques descriptives de la série de prix du pétrole futures NYMEX (en base quotidienne du 24 juin 1988 au 12 juin 2009)

\begin{tabular}{|l|r|}
\hline & \multicolumn{2}{|c|}{ WTI } \\
\hline Mean & 32.05290 \\
\hline Median & 21.37000 \\
\hline Maximum & 146.0800 \\
\hline Minimum & 9.640000 \\
\hline Std. Dev. & 23.67598 \\
\hline Skewness & 2.019925 \\
\hline Kurtosis & 7.137078 \\
\hline Jarque-Bera & 7408.817 \\
\hline Probability & 0.000000 \\
\hline Observations & 5318 \\
\hline
\end{tabular}

Les statistiques descriptives de la série de prix du pétrole NYMEX révèlent que la valeur moyenne du baril sur la période 1988-2009 est de \$32 US. Nous pouvons remarquer graphiquement cette tendance (Figure 1), que le changement structurel de mars-août 2008 vient perturber: le prix du baril de pétrole a ainsi atteint un maximum de \$146 le 3 juillet 2008, pour retomber à $\$ 30$ en décembre 2008, pour augmenter à nouveau à $\$ 70$ en 2009. De plus, nous remarquons que la série de prix du pétrole NYMEX n'est pas normalement distribuée, ce qui est caractéristique des séries temporelles financières.

Dans la Figure 2, nous détectons plus précisément l'origine du changement structurel à l'aide du test de racine unitaire avec rupture de tendance endogène de Zivot-Andrews (1992). Ce test détecte par estimation récursive la présence d'une racine unitaire dans la série, tout en autorisant une rupture de tendance à une date inconnue. D'après ce test, la date potentielle de changement structurel est le 22 août 2007. En appliquant ce test sur la transformation logarithmique du prix du pétrole, nous identifions une deuxième date potentielle de changement structurel le 25 septembre 2008 (à une période où le baril de pétrole se trouve aux alentours de US\$100). Par ailleurs, nous trouvons que la série de prix du pétrole est intégrée d'ordre $1(I(1))$. 


\section{Encadré 1 : Les mesures de la volatilité}

Le terme volatilité étant polysémique, les définitions suivantes sont couramment admises parmi les praticiens de la finance :

- Volatilité extraite d'un modèle $\operatorname{GARCH}(p, q)$ (Bollerslev (1986)) en fréquence quotidienne :

$$
\begin{aligned}
& Y_{t}=\theta X_{t}^{\prime}+\epsilon_{t} \\
& \sigma_{t}^{2}=\omega+\sum_{i=1}^{p} \alpha_{i} \epsilon_{t-i}^{2}+\sum_{j=1}^{q} \beta_{j} \sigma_{t-j}^{2}
\end{aligned}
$$

avec $\sigma_{t}^{2}$ la variance conditionnelle, qui est fonction du terme constant $\omega$, du coefficient ARCH $\varepsilon_{t-i}^{2}$, et du coefficient GARCH $\sigma_{t-j}^{2}$. L'estimation d'un modèle classique $\operatorname{GARCH}(1,1)$ sur la série WTI transformée en différences premières conduit au graphique:

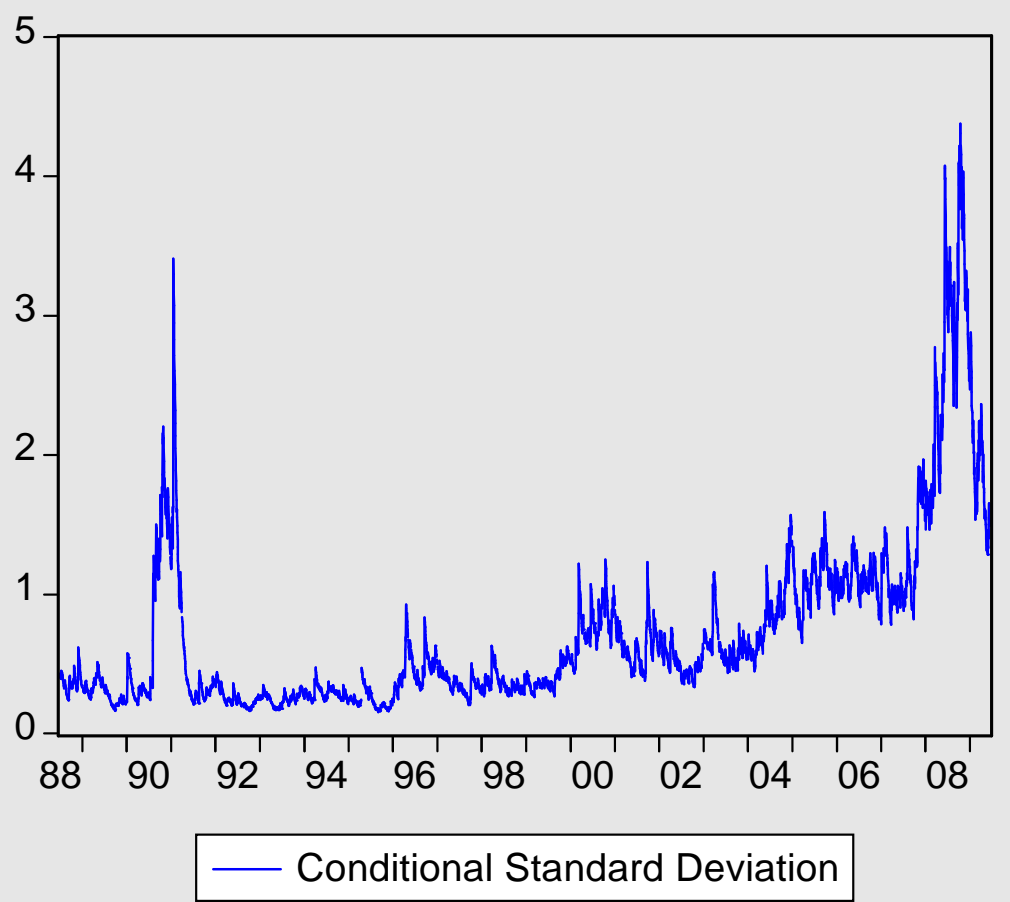

- Volatilité basée sur les écarts intra-quotidiens de prix entre l'ouverture et la fermeture du marché par Parkinson (1980) : 


$$
\hat{\sigma}_{P}^{2}=\frac{1}{4 n \ln 2} \sum_{i=1}^{n}\left(u_{i}-d_{i}\right)^{2}
$$

avec $\hat{\sigma}_{P}^{2}$ la volatilité de l'actif financier à mesurer, $n$ le nombre de périodes quotidiennes, $u_{t}$ la valeur de prix haute normalisée, $d_{t}$ la valeur de prix basse normalisée ;

ou celle de Garman et Klass (1980) qui est plus efficiente car elle repose sur l'hypothèse de variance minimale:

$\hat{\sigma}_{G K}^{2}=\frac{0.511}{n} \sum_{i=1}^{n}\left(u_{i}-d_{i}\right)^{2}-\frac{0.019}{n} \sum_{i=1}^{n}\left[c_{i}\left(u_{i}+d_{i}\right)-2 u_{i} d_{i}\right]-\frac{0.383}{n} \sum_{i=1}^{n} c_{i}^{2}$

avec $c_{t}$ le prix de clôture de la journée $t$.

- Volatilité extraite des prix d'options, obtenue en inversant numériquement la formule de Black-Scholes (1976) :

$$
\min _{\sigma}\left(C(\tau, K)_{a b s}-C(\tau, K, \sigma)_{B S}\right)^{2}
$$

avec $C(\tau, K)_{o b s}$ le prix observé de l'option d'achat (call), $C(\tau, K, \sigma)_{B S}$ le prix BlackScholes calculé en utilisant la volatilité implicite $\sigma$. Par définition, $C(\tau, K)_{\text {obs }}=C(\tau, K, \sigma)_{\text {BS. }}$.

$$
R V_{t+1}(\Delta) \equiv \sum_{j-1}^{1 / \Delta} r_{t+j \cdot \Delta, \Delta}^{2}
$$

avec $p_{t}$ le prix de l'actif considéré à l'instant $t, r_{t}$ le rendement, et $\Delta$ la période de temps telle que $r_{t, \Delta} \equiv p(t)-p(t-\Delta)$ 


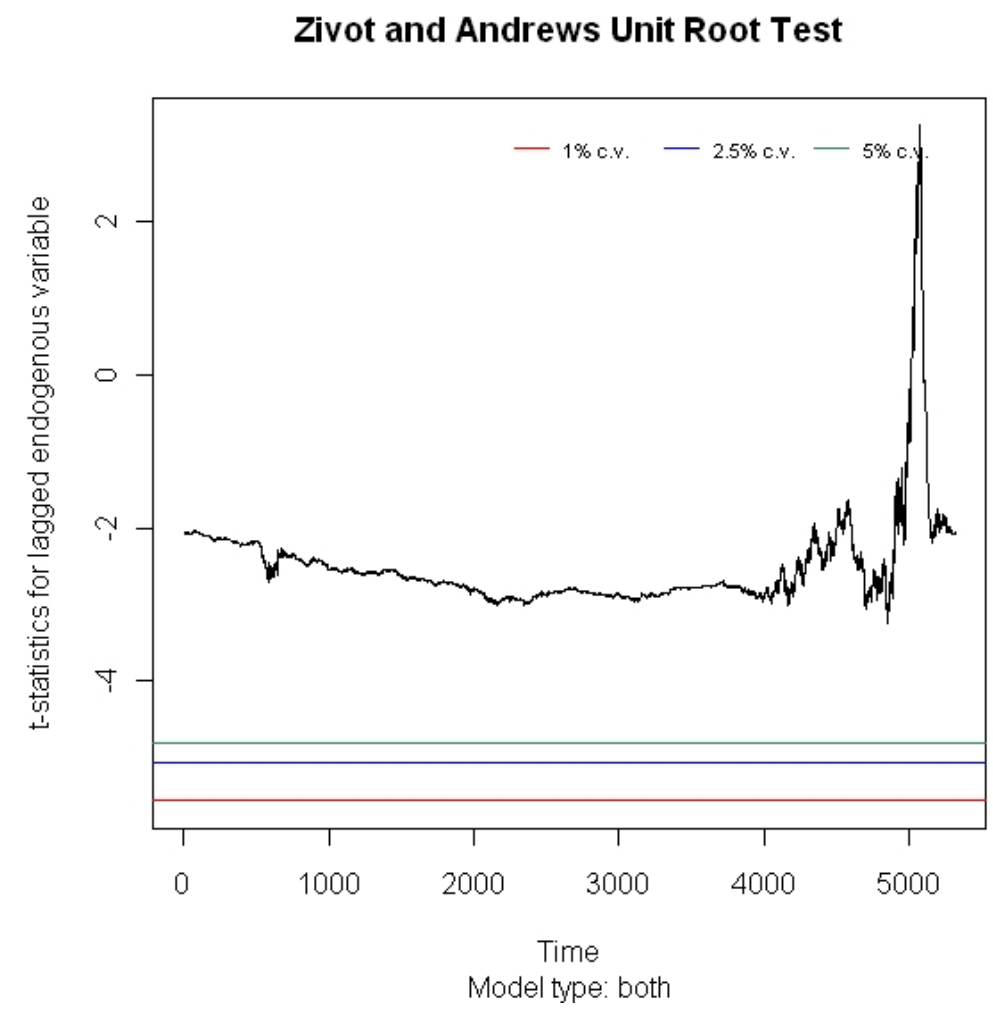

Figure 2 : Test de racine unitaire avec détection endogène de rupture de tendance de Zivot-Andrews (1992) appliqué à au prix du pétrole futures NYMEX en base quotidienne du 24 juin 1988 au 12 juin 2009

\section{Aux fondamentaux pourtant bien identifiés...}

Le prix mondial du pétrole se forme à partir du prix West Texas Intermediate (WTI) côté depuis 1978 au NYMEX, et plus marginalement du Brent. Des marchés spots physiques existent pour différents bruts, avec en lien des cotations de gré-à-gré, où le risque de contrepartie est beaucoup plus important. Il est raisonnable de penser que le prix mondial du pétrole se fixe sur les plates-formes d'échange électronique du Chicago Mercantile Exchange (CME, dont le NYMEX est une division) et de l'Intercontinental Futures Exchange (ICE) basé à Atlanta, proposant également un contrat très liquide sur le WTI, sous le contrôle réglementaire de la Commodities Futures Trading Commission (CFTC) américaine. Le déterminant principal du prix est l'anticipation que se font professionnels et investisseurs de ce que seront demain les équilibre entre offre et demande sur le marché du pétrole. 


\section{Encadré 2 : La notion de volatilité « excessive»}

La notion de volatilité « excessive» est difficile à mobiliser en finance, car elle sousentend que l'on puisse établir un scénario de référence par rapport auquel la fluctuation du cours d'un actif apparaît «normal». Dumas et al. (2006) examinent cette question à la lumière des marchés d'action aux Etats-Unis. Dans ce cadre de la finance comportementale, la fluctuation excessive d'un actif peut être causée par des «sentiments » de marché irrationnels. Mis à part la contribution initiale de Shiller (1981), il n'existe pas de corpus théorique expliquant quelle serait la meilleure réponse de la part d'investisseurs rationnels concernant l'allocation de leur richesse. Dumas et al. (2006) identifient une autre catégorie d'investisseurs: des investisseurs «irrationnels » qui seraient responsables de la volatilité «excessive» de l'actif considéré. En étudiant les performances historiques de l'actif, les investisseurs peuvent identifier des périodes de volatilité excessive au cours desquelles ils adapteront rationnellement leurs anticipations : on peut ainsi mettre en évidence des effets d'apprentissage (learning) rationnel (Timmermann (1993, 1996)). Ainsi, seuls les agents dont les anticipations sont les plus précises «survivent» (dans l'optique d'accumuler des richesses) dans le long terme. Dumas et al. (2006) concluent que les investisseurs rationnels écartent du marché au final les investisseurs irrationnels, mais que ce phénomène s'inscrit dans un processus de temps assez long.

\section{Canaux de transmission de chocs sur le marché du pétrole}

D’après Hamilton (1983, 1996), les chocs de prix du pétrole sont liés aux contexte macroéconomique global à travers :

1. l'état des stocks,

2. l'utilisation des capacités de production,

3. les indicateurs macroéconomiques (inflation, taux d'intérêt, bons au Trésor),

4. les disruptions dans l'approvisionnement physique en pétrole.

Hamilton soutient ainsi la thèse selon laquelle en présence de chocs sur le marché du pétrole (telles que les guerres du Golfe, entraînant des disruptions dans l'approvisionnement mondial en pétrole), la demande pour des indicateurs clefs de biens de consommation et d'investissement chute et entraîne une récession. 
D’après Blanchard et Gali (2007), les chocs sur le marché du pétrole des années 2000 (avant 2008) coïncident avec des chocs plus larges ayant eu lieu, par exemple, sur les autres marchés de matières premières. Les conséquences sont moins fortes sur les prix, les salaires, l'activité économique et l'emploi que dans les années 1970 grâce à une moindre rigidité du salaire réel.

Une autre raison peut être trouvée dans la plus grande crédibilité de la politique monétaire : la réponse de l'inflation anticipée aux chocs sur le marché du pétrole est beaucoup plus faible. Enfin, une raison pour expliquer la moindre incidence des chocs du marché du pétrole sur l'activité économique réside dans la diminution de la part du pétrole dans la consommation et la production.

Blanchard et Riggi (2009) révèlent deux autres changements dans la structure de l'économie qui peuvent avoir un effet explicatif sur la transmission des chocs du marché du pétrole: (i) la disparition de l'indexation des salaires, et (ii) l'amélioration de la crédibilité de la politique monétaire.

Hamilton (2008) distingue les facteurs explicatifs des chocs affectant le marché du pétrole sur la période 1970-1997 et ceux des années 2000. Durant 1970-1997, la faible élasticitéprix à la demande et l'offre de court-terme, la vulnérabilité aux disruptions dans l'approvisionnement en pétrole et le pic de la production de pétrole américaine peuvent être avancés comme les principaux facteurs explicatifs du comportement du prix du pétrole. Dans les années 2000, un profond changement de la demande provenant des pays nouvellement industrialisés et la reconnaissance de la dimension finie de la ressource pétrole semblent offrir une explication plausible aux récents développements du prix du pétrole. D'après Hamilton (2008), la rente de rareté de la ressource pétrole - chère aux économistes de l'environnement - pourrait ainsi devenir un élément d'explication central des évolutions futures du prix du pétrole.

Kilian (2008a) étudie la transmission des chocs exogènes du marché du pétrole sur l'économie américaine. Contrairement à Bernanke, Gertler et Watson (1997), il identifie une forte chute du PIB réel cinq trimestres après un choc exogène sur l'offre de pétrole, et un pic dans l'inflation du Consumer Price Index (CPI) trois trimestres après un choc. Plus que la production physique de pétrole, l'auteur souligne le rôle joué par les anticipations des agents concernant les craintes de futures ruptures d'approvisionnement en pétrole dans la formation de ces chocs. 


\section{Encadré 3 : Elasticité prix de la demande de pétrole}

L'élasticité-prix de la demande mesure le changement (en pourcentage) de la quantité demandée divisée par le changement (en pourcentage) du prix au fur et à mesure d'une courbe de demande. Hamilton (2008) documente que l'élasticité prix de la demande en essence est de $\mathbf{- 0 . 2 5}$ à court-terme et comprise entre $\mathbf{- 0 . 5 0}$ et $\mathbf{- 0 . 7 5}$ à long-terme. Si le pétrole brut représente la moitié du coût de l'essence mise en vente dans le commerce, une augmentation de $10 \%$ du prix du brut correspond ainsi à une augmentation de $5 \%$ du prix de l'essence. Dahl (1993) et Cooper (2003) estiment que l'élasticité de long-terme de la demande en pétrole brut oscille autour de $[-0.2 ;-0.3]$, tandis que l'élasticité de court-terme se trouve en-dessous de -0.1 .

La Banque d'Angleterre (BOE, 2008) a par ailleurs livré ses propres estimations, en comparaison avec les valeurs publiées par l'Agence Internationale de l'Energie :

Table 1 IEA price and income elasticity of oil demand

\begin{tabular}{lrrrrr} 
& $\begin{array}{r}\text { Advanced } \\
\text { economies }\end{array}$ & $\begin{array}{r}\text { Developing } \\
\text { Asia }\end{array}$ & $\begin{array}{r}\text { Oil } \\
\text { exporters }\end{array}$ & $\begin{array}{r}\text { Latin } \\
\text { America }\end{array}$ & $\begin{array}{r}\text { Rest of } \\
\text { the world }\end{array}$ \\
\hline $\begin{array}{l}\text { Price elasticity } \\
\text { Short run }\end{array}$ & -0.02 & -0.03 & 0.05 & -0.03 & -0.03 \\
Long run & -0.12 & -0.11 & -0.25 & -0.21 & -0.28 \\
Income elasticity & & & & & \\
Short run & 0.04 & 0.14 & 0.08 & 0.09 & 0.09 \\
Long run & 0.22 & 0.49 & 0.39 & 0.73 & 0.94 \\
& & & & & \\
Note: Based on annual data from 1979 to 2005. & & & & \\
Source: IEA World Energy Outlook, 2006. & & & & \\
\end{tabular}

Table 2 Bank price and income elasticity of oil demand

\begin{tabular}{lrrrrr} 
& $\begin{array}{c}\text { Advanced } \\
\text { economies }\end{array}$ & $\begin{array}{l}\text { Developing } \\
\text { Asia }\end{array}$ & $\begin{array}{r}\text { Oil } \\
\text { exporters }\end{array}$ & $\begin{array}{r}\text { Latin } \\
\text { America }\end{array}$ & $\begin{array}{r}\text { Rest of } \\
\text { the world }\end{array}$ \\
\hline $\begin{array}{l}\text { Price elasticity } \\
\text { Short run }\end{array}$ & -0.02 & -0.04 & 0.01 & -0.11 & -0.05 \\
Long run & -0.12 & -0.13 & 0.04 & -0.24 & -0.06 \\
$\begin{array}{l}\text { Income elasticity } \\
\text { Short run }\end{array}$ & & & & & \\
Long run & 0.05 & 0.17 & 0.09 & 0.16 & 0.13 \\
& 0.27 & 0.56 & 0.50 & 0.33 & 0.16 \\
& & & & & \\
Source: Bank calculations. & & & & &
\end{tabular}

Source : BOE (2008) 
Kilian (2008b) compare les effets de chocs exogènes dans la production mondiale de pétrole sur l'activité économique et l'inflation des pays du G7. Ses résultats indiquent qu'une disruption dans l'approvisionnement en pétrole entraîne une réduction temporaire du PIB réel, l'effet étant plus fort lors de la seconde année après le choc. Les réponses du taux d'inflation sont plus diverses, et leur pic se trouve après trois/quatre trimestres. L'auteur montre que des chocs exogènes en approvisionnement en pétrole ne génèrent pas nécessairement une situation d'inflation ou de stagflation. Ces effets peuvent également transiter par une baisse du salaire réel, des taux d'intérêt de court-terme plus élevés, et une dépréciation de la monnaie nationale par rapport au dollar.

Kilian (2009) estime les effets dynamiques de trois types de chocs sur le prix réel du pétrole : (i) des chocs sur l'offre de pétrole, (ii) des chocs sur la demande globale de matières premières dans l'industrie, et (iii) des chocs de demande spécifiques au marché du pétrole (tels qu'une demande de précaution plus élevée liée à des craintes de raréfaction future des ressources pétrolières). Cette étude révèle des changements dans la composition des chocs affectant le marché du pétrole. Le principal résultat souligne que les accroissements de prix du pétrole (hors crise spéculative) sont liés principalement à des chocs de demande globale, et non à des disruptions dans l'approvisionnement en pétrole ou à une demande de précaution pour les ressources pétrolières non anticipée.

Kilian (2010) analyse plus précisément les déterminants de la demande en essence aux États-Unis. Il identifie parmi ces déterminants l'influence $(i)$ des chocs de demande positifs et répétés sur les marchés globaux de matières premières (dans la mesure où les économies développées ont connu une croissance forte et que les pays émergents s'intègrent progressivement dans l'économie globale), et (ii) des chocs de demande spécifique au marché du pétrole et ceux affectant l'offre dans le secteur américain de la raffinerie. A partir de mai 2008, le renversement des anticipations concernant les prévisions de conjoncture économique a conduit a une forte chute des prix de l'essence, suite à la forte chute dans la demande de pétrole qui a perduré en 2008. Kilian (2010) souligne donc essentiellement que les causes des augmentations et des chutes de prix de l'essence aux Etats-Unis peuvent être d'origine différente. L'explication de telles asymétries fait l'objet de recherches en cours. 
Selon Dvir et Rogoff (2009), il apparaît essentiel d'adopter une vision de long terme pour comprendre les effets dynamiques de chocs du marché du pétrole sur l'activité économique. Les chocs sur le marché du pétrole ont des effets très différents sur le prix réel du pétrole, non en vertu de leur origine (offre ou demande), mais selon l'(in)capacité des acteurs de marché à restreindre l'accès à l'offre de pétrole. Lorsque des restrictions existent sur l'accès aux excès d'offre, les chocs sur le marché du pétrole génèrent des chocs sur les niveaux de prix à la fois persistant et très volatiles. Sans ces restrictions, des chocs sur le marché du pétrole sont rapidement absorbés, et ne conduisent pas forcément à une plus grande persistance ou volatilité du prix.

\section{Remis en cause par la crise de 2007-2008.}

\section{Les réformes institutionnelles des années 2000}

La CFTC (2008) définit par agent non-commercial un trader qui n'utilise pas les contrats futures de pétrole pour couvrir une position. Les agents cherchant à couvrir leur position sont typiquement des producteurs et des consommateurs de la matière première physique, qui utilisent les contrats futures pour se prémunir contre le risque de changement de prix. Les agents non-commerciaux eux recherchent le profit en prenant des positions sur le marché futures, et en espérant retirer des gains des changements de prix de la matière première, mais ne sont pas intéressés par sa livraison physique.

Medlock et Jaffe (2009) rapportent que cette dernière catégorie d'agents -suspectés d'être les «spéculateurs » - a connu une augmentation beaucoup plus forte de son activité sur les marchés futures de pétrole aux États-Unis ${ }^{2}$ que la catégorie traditionnelle des acteurs de marché «physiques ». En effet, les agents non-commerciaux représentent plus de $50 \%$ des agents possédant une position ouverte ${ }^{3}$ sur les marchés futures du pétrole, contre $20 \%$ avant 2002. L'entrée des agents non-commerciaux sur les marchés pétrole peut ainsi expliquer le fort accroissement des positions ouvertes sur les marchés futures du pétrole. Cette entrée est par ailleurs fortement corrélée avec l'accroissement des prix du pétrole. Ces changements sont dus d'après Medlock et Jaffe (2009) aux réformes institutionnelles des années 2000 aux Etats-Unis, et plus précisément au Commodity Future Modernization Act (CFMA).

\footnotetext{
${ }^{2}$ A cela peuvent s'ajouter des stratégies de trading associant prix du pétrole et taux de change du dollar. En 2008, les importations de pétrole aux États-Unis représentaient \$331 milliards, soit 47\% du déficit commercial (contre
} 
D'après l'U.S. Government Accountability Office (2007), cette nouvelle législation a permis aux agents financiers de s'affranchir des limites de spéculation, et a rendu plus difficile la tâche de la CFTC concernant la régulation des marchés futures du pétrole. D'après la CFTC (2008), seules les positions dénommées «bona fide hedges » sont exemptes de limites de transactions. Nous détaillons les suites données à ces exemptions dans la section suivante. Le CFMA, approuvé par le Congrès américain le 15 décembre 2000 et signé par le Président Clinton le 21 décembre 2000, instaure une législation plus flexible que par le passé, permettant à des agents financiers nouveaux (tels que les fonds d'index de matières premières et les agents «swappant» (échangeant) des matières premières) d'accéder aux marchés dérivés de pétrole. Ainsi, certaines transactions sur les marchés dérivés de pétrole se sont retrouvées en-dehors de la juridiction de la CFTC (voir Medlock et Jaffe (2009) pour plus de détails). Il est important de noter également que le CFMA a retiré les transactions «swap» des limites de spéculation établies par la CFTC, et favorisé l'émergence de «contrats de différences» (voir Medlock et Jaffe (2009) pour plus de détails).

\section{La connexion progressive entre contrats futures de différentes maturités et le développement des traders financiers}

Büyükşahin et al. (2008) identifient une rupture structurelle dans la relation entre les contrats futures du marché du pétrole de différentes maturités qu'ils associent à des changements :

- de fondamentaux,

- dans la structure par terme,

- dans la composition des traders opérant sur le marché futures.

Basé sur une base de données unique en terme de positions des traders sur les marchés options et futures de pétrole, leurs résultats illustrent le rôle joué par l'accroissement de l'activité des agents «swappant » (échangeant) des matières premières, par les hedge funds et d'autres traders financiers, dans le lien entre contrats futures de différentes maturités.

19\% en 2002). La corrélation entre le prix du pétrole et la valeur du dollar est passée de 0.08 durant 1986-2000 à 0.82 durant 2001-2009.

${ }^{3}$ La position ouverte se définit comme le nombre de contrats ouverts caractérisés comme « actifs » à l'instant $t$. 
La contribution de Büyükşahin et al. (2008) illustre sans doute le mieux le rôle joué par les traders dans possible formation d'une «bulle » spéculative entre mars et août 2008. Pour comprendre l'ampleur de cette crise, les contrats WTI de pétrole (crude oil) enregistraient une position ouverte de $\$ 322$ milliards sur le NYMEX fin août 2008. Ce chiffre correspond à une multiplication par quinze des positions ouvertes sur les marchés dérivés de pétrole (futures et options) entre 2000 et 2008. Cette situation peut s'expliquer : 1) par un rallongement considérable de la structure par terme des contrats futures (avec la connexion progressive entre contrats futures de différentes maturités, alors que jusqu'en 2001-2004 les contrats futures de pétrole étaient valorisés et échangés comme sur des marchés segmentés), 2) par des changements dans la composition des traders opérant sur leur activité sur les marchés pétrole.

Concernant ce dernier point, Büyükşahin et al. (2008) utilisent dans leur étude une base de données non publique de la Commodity Futures Trading Commission's (CFTC) américaine enregistrant les positions individuelles quotidiennes des traders selon plusieurs maturités de contrats futures WTI, et selon une classification par type d'activité. Parmi celles-ci, on peut distinguer les catégories :

- « commerce traditionnel»: producteurs, manufacturiers, intermédiaires, marchands,

- «non commerciales » : brokers, traders, hedge funds, participants non-enregistrés,

- agents «swappant » (échangeant) des matières premières ${ }^{4}$.

Ainsi, les liens entre contrats futures de maturité à un et deux ans ont été développés par les traders financiers (dont les hedge-funds), tandis que les agents «swappant» des matières premières ont assuré le lien entre les contrats les plus proches (ce qui constitue leur cœur de métier). La participation des traders financiers et des agents « Swappant » des matières premières sur le marché du pétrole a ainsi contribué à l'accroissement de l'activité de marché sans précédent de mars-août 2008 (voir Figures 3,4,5), qui s'est ensuite effondrée suite à une baisse de la demande et une augmentation simultanée des capacités de production de réserve (spare capacity).

\footnotetext{
4 Ces derniers effectuent des «swaps» de transaction de gré-à-gré avec des entités commerciales, des fonds indexant diverses matières premières, ou d'autres investisseurs financiers.
} 
Les conclusions de Büyükşahin et al. (2008) sont étayées par les faits suivants : (i) les positions ouvertes à plus d'un an ont crû deux fois plus rapidement que les positions ouvertes sur des maturités plus courtes entre 2000 et 2008, (ii) la part de marché des agents «swappeurs » de matières premières a crû de façon exponentielle dans les années 20022003 sur fond de boom des investissements dans les index de matières premières, (iii) la part de marché des « commerçants traditionnels » a diminué de moitié depuis 2000, tandis que les traders financiers étendaient leur activité sur le marché pétrole 5 .

Cette analyse est partagée par d'autres études, parmi lesquels nous pouvons citer la Banque d'Angleterre (BOE, 2008), Hamilton (2009), Parsons (2009), Tang et Xiong (2009).

Le rapport "What can be said about the rise and fall in oil prices?" (BOE, 2008), préparé par Saporta, Trott et Tudela, consiste en une analyse statistique complète et récente des causes potentielles de la crise de mars-août 2008 sur le marché du pétrole. Les auteurs utilisent les données publiques de la CFTC sur la position longue des agents non-financiers pour mettre en œuvre leur propre analyse statistique (basée sur des modèles vectoriels autorégressifs) sur les échantillons 2003-2006 et 2006-2008 du prix du pétrole. Ils concluent de façon très prudente qu'il n'est pas possible d'éliminer complètement l'hypothèse de bulle spéculative (engendrée par les agents non-commerciaux) dans l'explication de la dynamique du prix du pétrole en 2008 , et mettent en avant les limites inhérentes à l'utilisation des données publiques de la CFTC. Saporta, Trott et Tudela (BOE, 2008) soulignent donc très clairement l'intérêt d'accéder aux données confidentielles de la CFTC $^{6}$ sur les positions des investisseurs, permettant une identification plus précise des flux émanant des «spéculateurs », auxquelles Büyükşahin et al. (2008) ont eu accès pour mener l'étude détaillée précédemment.

Hamilton (2009) explore les causes et les conséquences du choc ayant impacté le marché du pétrole en 2007-2008. Il oppose pour cela les précédents chocs historiques dus à des disruptions physiques dans l'approvisionnement en pétrole, et la forte augmentation des prix enregistrée en 2007-2008 dans un contexte de forte demande et de production mondiale stagnante. Hamilton met l'accent sur le rôle de la spéculation dans l'explication de ce choc sur le prix du pétrole, et la forte chute des prix qui s'ensuit.

\footnotetext{
${ }^{5}$ La part de marché des traders financiers a plus que doublé, passant de moins de $20 \%$ à plus de $40 \%$ des positions ouvertes en futures et options entre 2000 et 2008.

6 Par ailleurs, Saporta, Trott et Tudela (BOE, 2008) mentionnent une note du Cabinet Office (2008) Britannique, qui suggère qu' "en l'absence de larges flux financiers sur les marchés futures du pétrole, il est possible que les prix n'auraient pas connu une telle ampleur haussière puis baissière en 2008 ».
} 
Cet épisode ressemble à la formation d'une bulle spéculative : les investisseurs achètent du pétrole non en tant que matière première mais en tant qu'actif financier. En prenant une position longue sur les contrats futures et en les revendant quelques semaines avant leur expiration, une stratégie spéculative consiste à réutiliser ces gains sur une séquence des positions longues (toujours pour des contrats futures proches de leur date d'expiration). Lorsque les prix des matières premières augmentent, le prix de vente est supérieur au prix d'achat, ce qui explique pourquoi cette séquence est bénéfique à l'investisseur. Celui-ci accumule en effet des positions longues synthétiques sur le marché du pétrole, sans jamais avoir à se préoccuper de la délivrance physique des contrats. Lorsque l'achat de contrats futures excède la revente des contrats arrivés à expiration, cette «financiarisation » du marché du pétrole conduit à la formation d'une bulle spéculative, avec un accroissement du prix futures et du prix spot sous-jacent.

Tang et Xiong (2009) examinent le processus de «financiarisation » des marchés de matières premières précipité par la croissance rapide des index d'investissement dans les matières premières depuis les années 2000. Les auteurs mettent en évidence la relation entre la présence croissance des investisseurs sur de tels index, et les chocs auxquels ont été soumis les marchés de matière première, au premier rang desquels se trouve le marché du pétrole. Les 2 indices les plus populaires, le Goldman Sachs Commodity Index (GSCI) et le Dow-Jones AIG Commodity Index (DJ-AIG), sont caractéristiques de ces mouvements prononcés sur les marchés de matières premières. Tang et Xiong (2009) révèlent que les interactions entre les marchés financiers et les marchés de matières premières deviennent de plus en plus importantes, et que la récente crise financière a largement contribué à augmenter la volatilité du prix des matières premières en 2008. Cette analyse est très documentée. Par exemple, Tang et Xiong (2009) révèlent que la valeur totale des échanges de matières premières par l'intermédiaire des fonds d'index pour les investisseurs institutionnels est passée de \$15 milliards en 2003 à \$200 milliards mi-2008. Basé sur un modèle de régression linéaire, les auteurs montrent tout d'abord que l'exposition d'index tel que le GSCI à des chocs liés aux marchés d'actions et au taux de change du dollar U.S. a significativement augmenté après 2004. Ils vérifient ensuite dans un modèle avec régressions roulantes que l'exposition croissante de secteurs énergie tel que le pétrole à ces mêmes chocs (marché d'actions, taux de change U.S.\$) est reliée de façon statistiquement significative à la présence des fonds d'index de matières premières. 
Puis, les auteurs analysent la relation entre les prix des matières premières et les flux d'investissement dans les fonds d'index. Leur analyse, basée sur un modèle vecteur autorégressif et les fonctions d'impulsion réponse associées, démontre le très fort pouvoir prédictif des flux d'investissement dans les fonds d'index en fonction d'autres variables liées aux marchés financiers et aux marchés de matières premières. Ce dernier résultat illustre plus précisément l'intégration croissante des 2 sphères. Enfin, les auteurs s'attachent à expliquer la volatilité du prix du pétrole à partir de l'activité des fonds d'index de matières premières. Les résultats de leurs régressions illustrent de façon statistique le lien entre le fort accroissement de la volatilité sur les marchés de matières premières en 2008 (dont le prix du pétrole) et l'activité des fonds d'index, donnant lieu à des effets de contagion.

\section{Figure 3: Number of Large Traders}

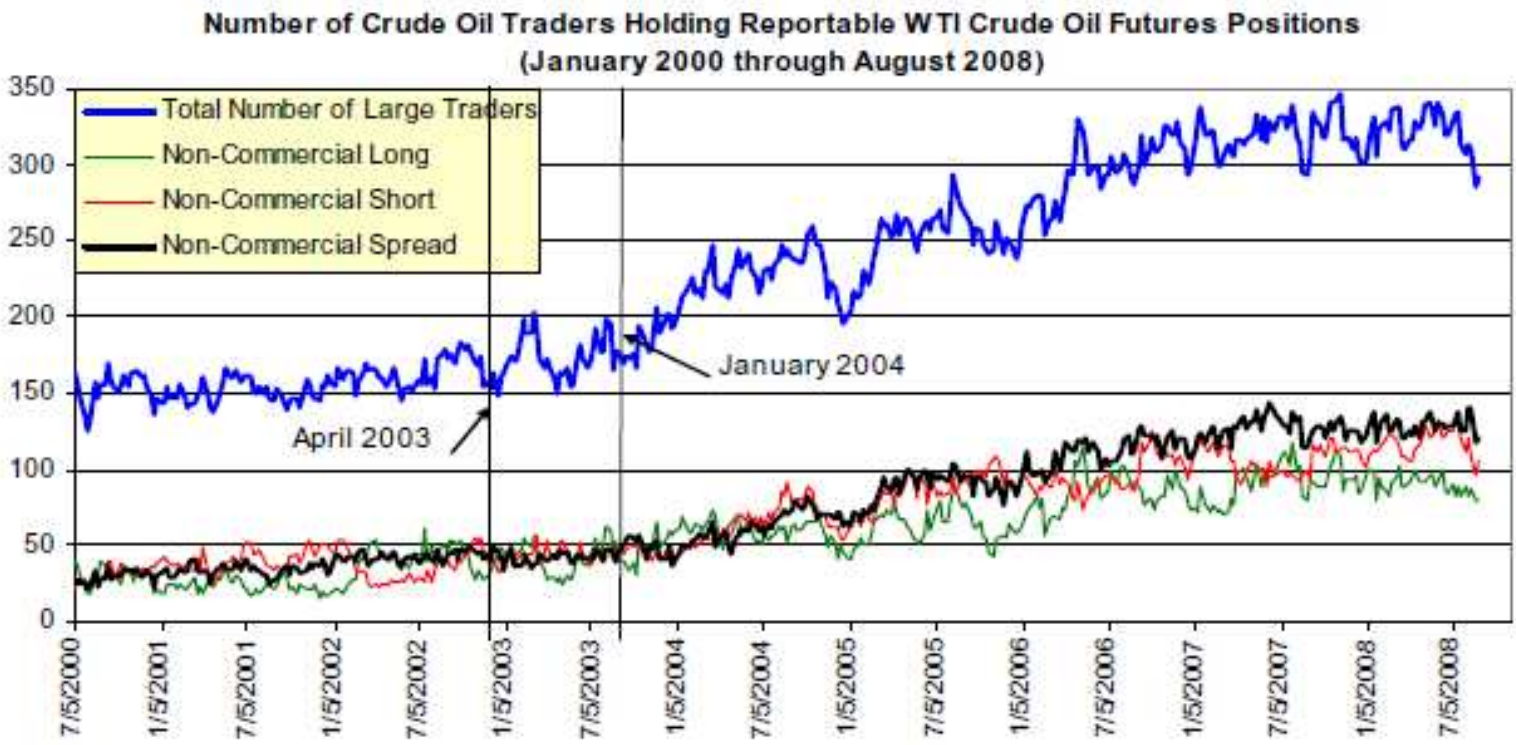

Notes: Figure 3 shows the total number of NYMEX traders reporting positions to the CFTC for its weekly Commitment of Traders Reports, between July 5.2000 and August 262008 (blue time series). The figure provides details for non-commercial traders, showing the number of non-commercials holding long (green time series), short (red time series) or spread positions (black time series). The number of non-commercial spread traders doubled between May 2001 and January 2004, and tripled again between February 2004 and July 2007.

Source: Büyükşahin et al. (2008). 


\section{Figure 4: Market Shares}

\section{Contribution to the WTI Futures Open Interest: All Maturities (July 2000 - August 2008)}

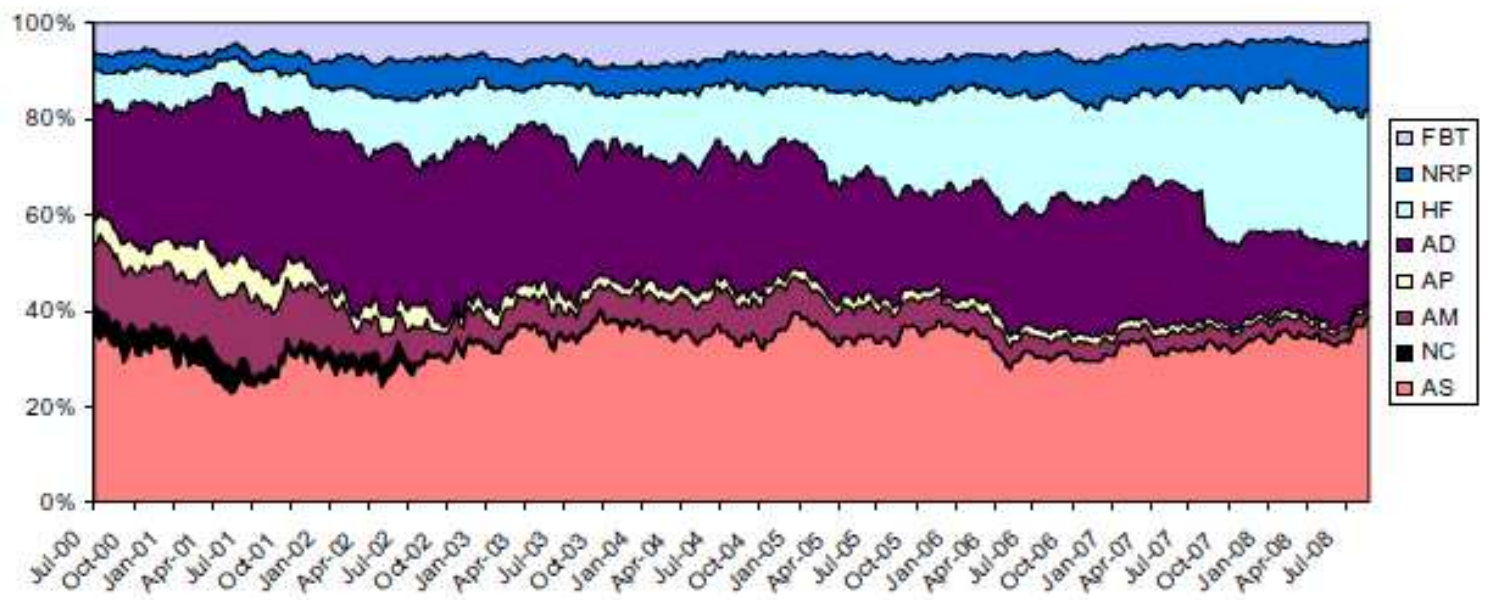

Notes: Figure 4 compares the contributions to the WTI futures open interest of eight types of large traders between July 52000 and August 26 2008. Commercial types include "Dealers/Merchants" (AD), "Manufacturers" (AM), "Producers" (AP), and "Commodity Swaps/Derivatives Dealers" (AS). Non-commercial types are "Hedge Funds" (HF), "Floor Brokers \& Traders" (FBT), and "Non-Registered Participants" (NRP). Prior to August 2003, the "NC" category sums the positions of presently inactive commercial traders.

Source : Büyükşahin et al. (2008).

Figure 5a: Daily Open Positions of the Major Commercial Trader Types, Julv 2000 to August 2008
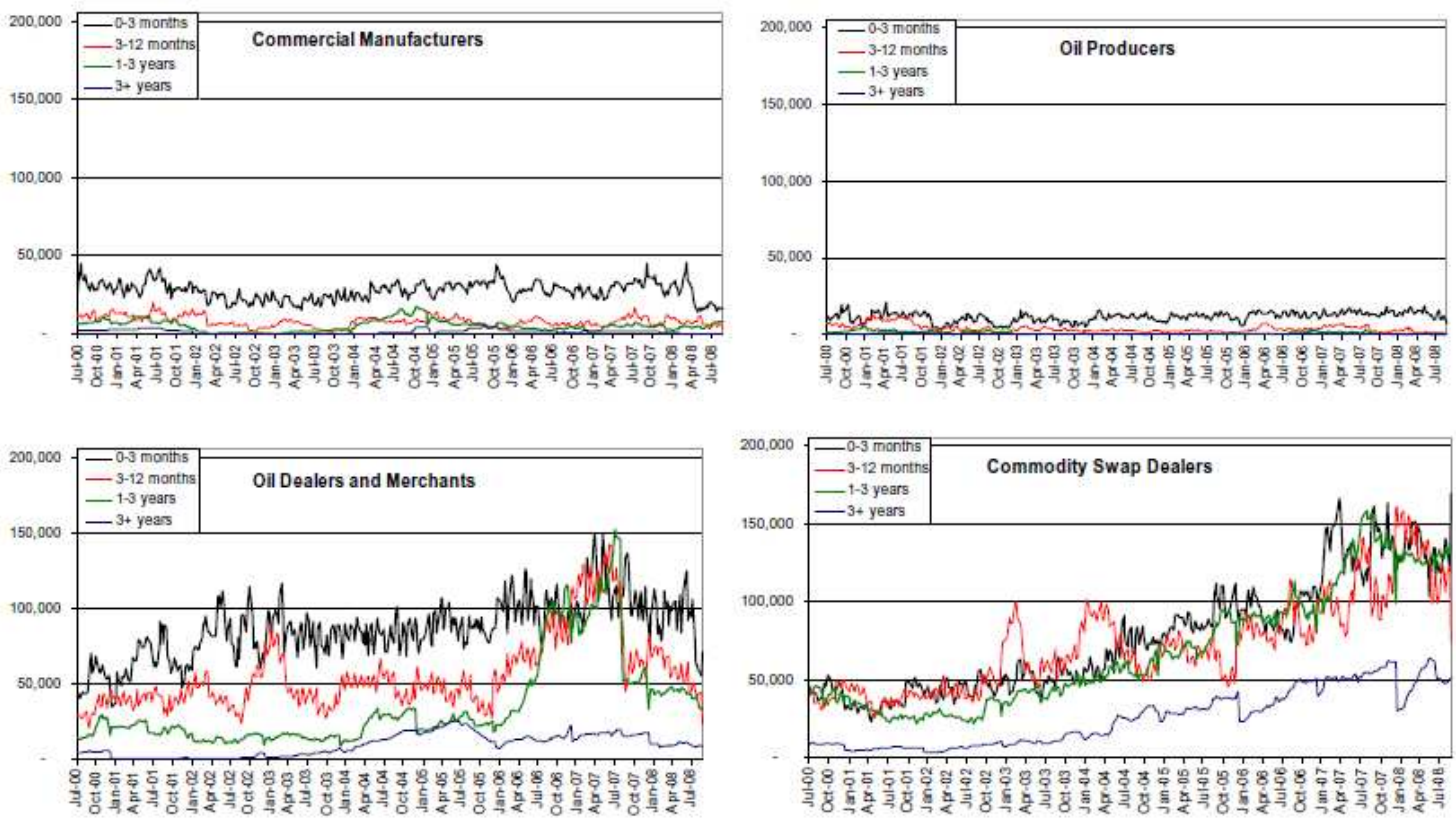

Notes: Figure 5a shows the open positions, by contract maturity, of the four most important commercial crude oil futures trader types: $A D=D e a l e r / M e r c h a n t$, $\mathrm{AM}=$ Manufacturer, $\mathrm{AP}=$ Producer, and $\mathrm{AS}=$ Commodity Swaps $/$ Derivatives Dealer (which includes $\mathrm{FS}=$ Financial Swaps $/$ Derivatives Dealer and $\mathrm{FA}=$ Arbitrageur or Broker/Dealer). Every Tuesday between July 2000 and August 2008, for each trader and each futures delivery month, we measure the trader's open interest in that contract as the average of the trader's long and short positions. The open interests of all reporting traders in a given category are then summed up appropriately (e.g., for all contracts with less than 3 months until maturity, etc.), and the Tuesday figures are then plotted. The graphs are scaled to allow for easy comparisons of the relative sizes of the open positions held by different trader categories. Figure $5 \mathrm{a}$ is directly comparable to Figure $5 \mathrm{~b}$ below.

Source : Büyükşahin et al. (2008). 

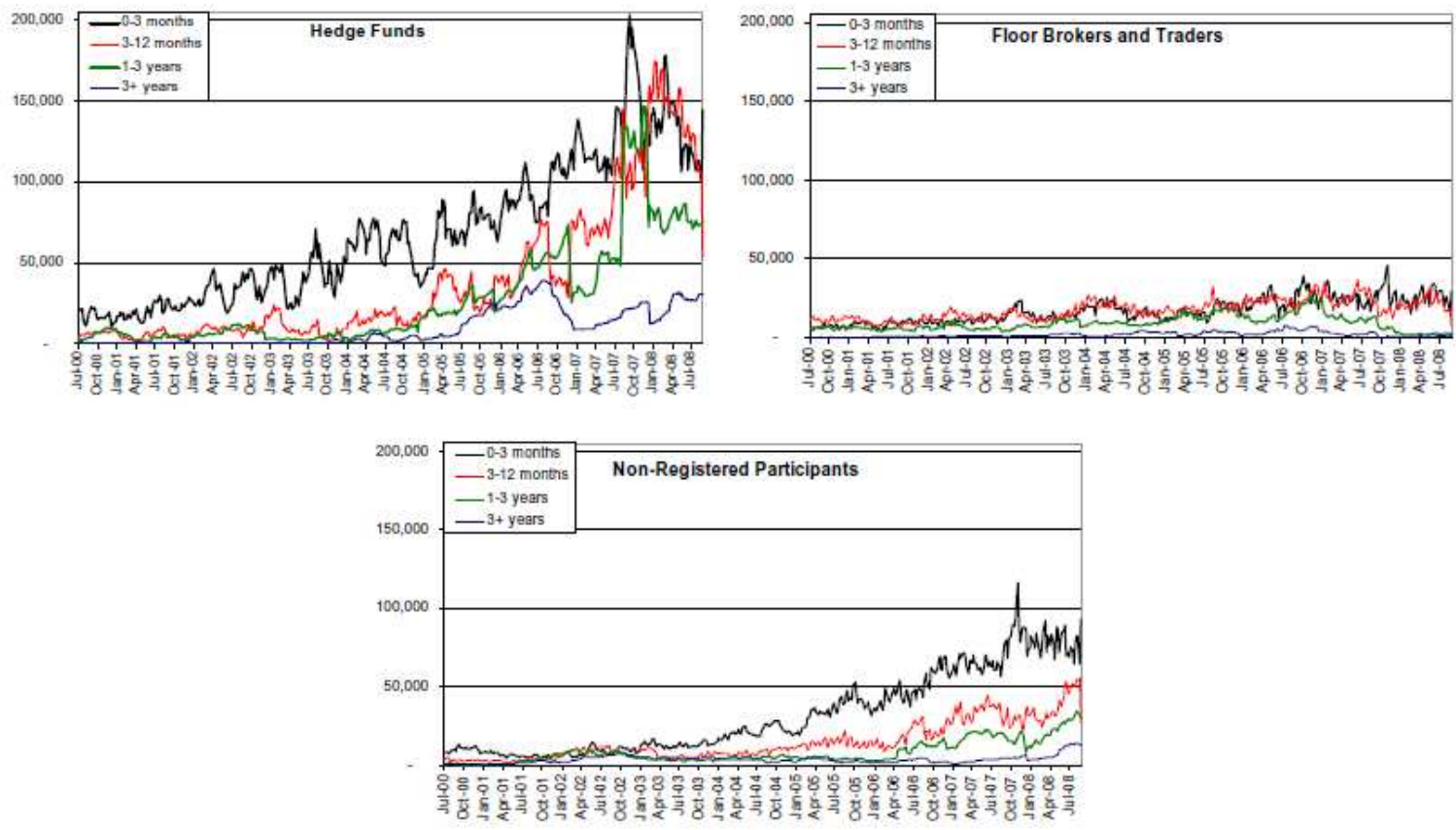

Notes: Figure 5b shows the open positions, by contract maturity, of the three most important non-commercial crude oil futures trader types: HF = Hedge Fund, and FBT = Floor Broker/Floor Trader. Every Tuesday between July 2000 and August 2008, for each trader and each futures delivery month, we measure the trader's open interest in that contract as the average of the trader's long and short positions. The open interests of all reporting traders in a given category are then summed up appropriately (e.g., for all contracts with less than 3 months until maturity, etc.), and the Tuesday figures are then plotted. The graphs are scaled to allow for easy comparisons of the relative sizes of the open positions held by different trader categories. Figure $5 \mathrm{~b}$ is directly comparable to Figure 5 a above.

Source : Büyükşahin et al. (2008).

Un manque de transparence concernant les échanges de produits dérivés hors places de marché et le contrôle des « swap dealers »

Parsons (2009) fournit des statistiques détaillées sur la position ouverte en contrats futures pétrole NYMEX, qui confirment l'analyse de Büyükşahin et al. (2008). De plus, il souligne que l'activité des «swap traders» de matières premières ne passe pas forcément par des places de marché telle que le NYMEX et ne sont pas forcément enregistrées par la CFTC, ce qui conduit à sous-estimer la vraie taille de la position ouverte sur les marchés futures de pétrole. Ses estimations, étayées par celles de la Bank for International Settlements (BIS, 2009), ne permettent cependant pas de fournir des informations plus précises. Le Staff Report on Commodity Swap Dealers and their positions de la CFTC (CFTC, 2008) n'a par ailleurs pas été rendu public. Parsons (2009) reporte que la CFTC n'a pas fixé de limites aux «swap traders » quant à leur position ouverte sur les marchés futures pétrole7.

\footnotetext{
${ }^{7}$ Cette exemption est en discussion depuis le 24/03/09 d'après la communication de la CFTC intitulée "Concept Release on Whether To Eliminate the Bona Fide Hedge Exemption for Certain Swap Dealers and Create a New Limited Risk Management Exemption From Speculative Position Limits".
} 
A partir de l'étude de Büyükşahin et al. (2008), l'auteur estime que la position ouverte moyenne des agents commerciaux a augmenté de 63\% au cours des 8 dernières années, tandis que celle des agents non commerciaux («swap dealers », hedge funds, autres agents non commerciaux) a augmenté de près de $\mathbf{6 0 0 \%}$. Toujours à partir de l'analyse de Büyükşahin et al. (2008), Parsons (2009) estime que la position ouverte des agents commerciaux sur les contrats de maturité longue ( 3 années ou plus) a augmenté de $72 \%$ au cours des 8 dernières années, tandis que celle des agents non-commerciaux a augmenté de plus de $1200 \%$. A partir de la Figure 4 présentée ci-dessus, Parsons (2009) remarque que les agents non-commerciaux représentent plus de $90 \%$ des positions ouvertes sur les marchés futures de pétrole en 2008 (dont 59\% pour les «swap dealers » et $24 \%$ pour les hedge funds).

\section{Le succès croissant des index de matières premières}

En s'appuyant sur la méthodologie de Masters et White (2008), Parsons (2009) estime que $\$ 130$ milliards (soit 64\%) des positions ouvertes sur les marchés futures de pétrole (NYMEX + ICE) auraient transité par les index de matières premières Goldman Sachs Commodity Index (GSCI) et Dow Jones-AIG Commodity Index (DJ-AIGCI) mi-2008 ${ }^{8}$. La CFTC (2008) fournit des estimations beaucoup plus faibles (\$51 milliards pour le seul NYMEX), mais ne documente ni sa méthodologie, ni les données utilisées pour effectuer ces estimations. D'après les rapports des index de matières premières, Parsons (2009) note que ceux-ci ont également décalé leur exposition sur les marchés futures de pétrole vers des maturités de contrats plus longues.

\section{Les agents non-commerciaux au cour de la hausse des prix du pétrole}

D'après Medlock et Jaffe (2009), la position ouverte des agents non-commerciaux a atteint $55 \%$ du total des positions ouvertes à son maximum en 2008, ce qui a coincidé avec le pic de prix du pétrole. Leur activité est notamment caractérisée par l'échange d'écarts de prix entre différents contrats («spread trading ») comme l'illustre la Figure 6.

\footnotetext{
${ }^{8}$ Voir également la déclaration de Masters (2008) devant le Sénat Américain.
} 
Figure 6 : Position ouverte des agents-non commerciaux par type de contrats

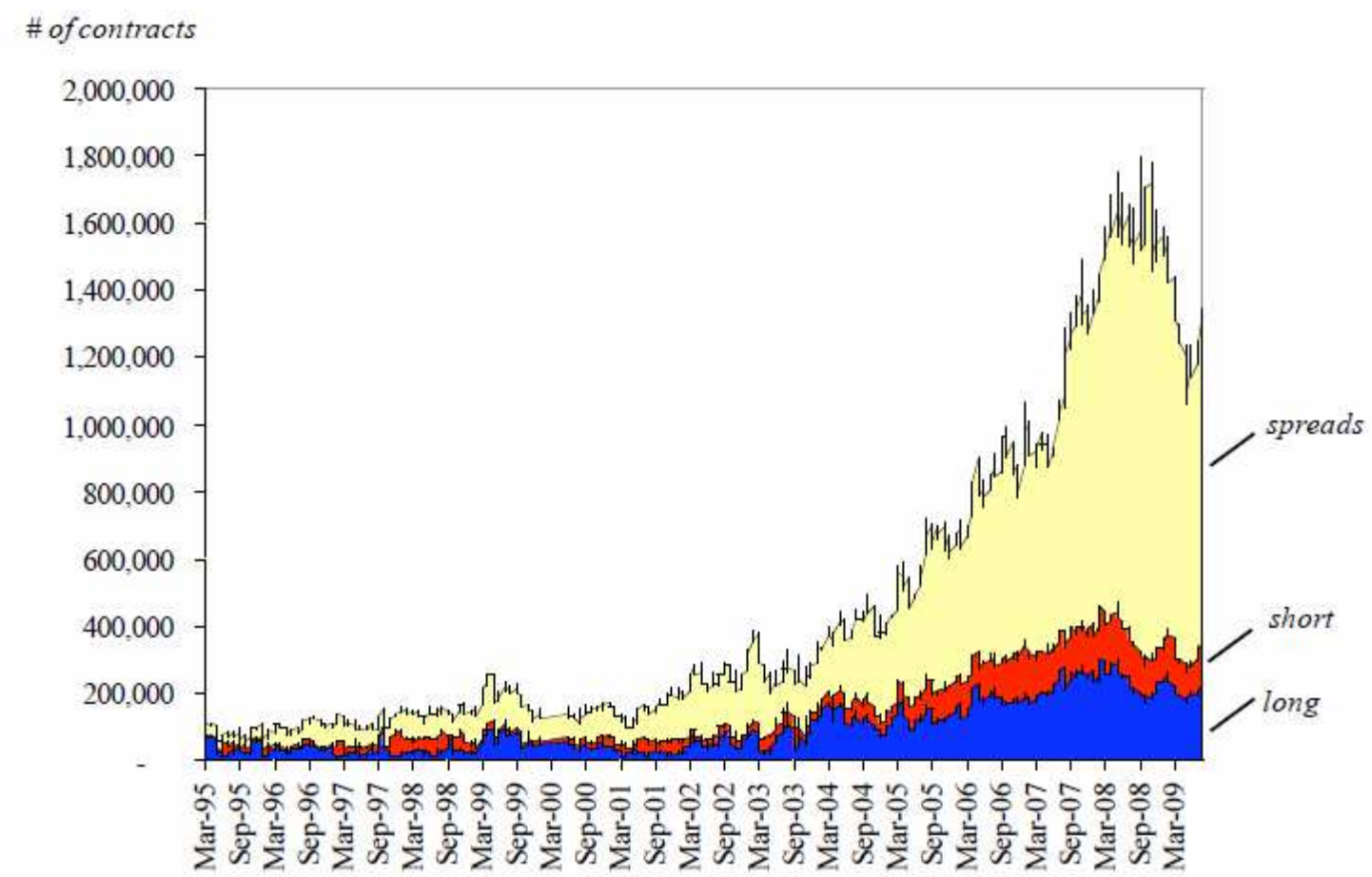

Source: CFTC Commitment of Traders Reports

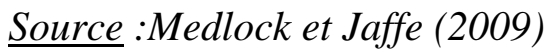

Entre 2006 et 2008, Medlock et Jaffe (2009) rapportent que la position ouverte des agents non-commerciaux est devenue un indicateur leader de la formation du prix. Cette situation a pu conduire à une «prophétie auto-réalisatrice» d'une hausse continue du prix du pétrole, au moins sur une courte période de temps. Enfin, leur position ouverte coïncide de façon remarquable avec l'évolution du prix du pétrole lui-même, comme nous pouvons le voir dans la Figure 7.

Medlock et Jaffe (2009) reportent que la CFTC ne prend en compte ni les changements de composition des traders opérant sur le marché, ni les changements d'anticipations agrégées des agents, pour déterminer des variations anormales du prix du pétrole, ou si le comportement d'un groupe particulier de trader peut s'avérer problématique. Les auteurs concluent qu'il est impossible d'ignorer les tendances dans les positions ouvertes des agents non-commerciaux et la variabilité du prix du pétrole, qui ont atteint des niveaux records depuis les réformes institutionnelles du CFMA. 
Figure 7 : Position ouverte des agents-non commerciaux et prix du pétrole WTI

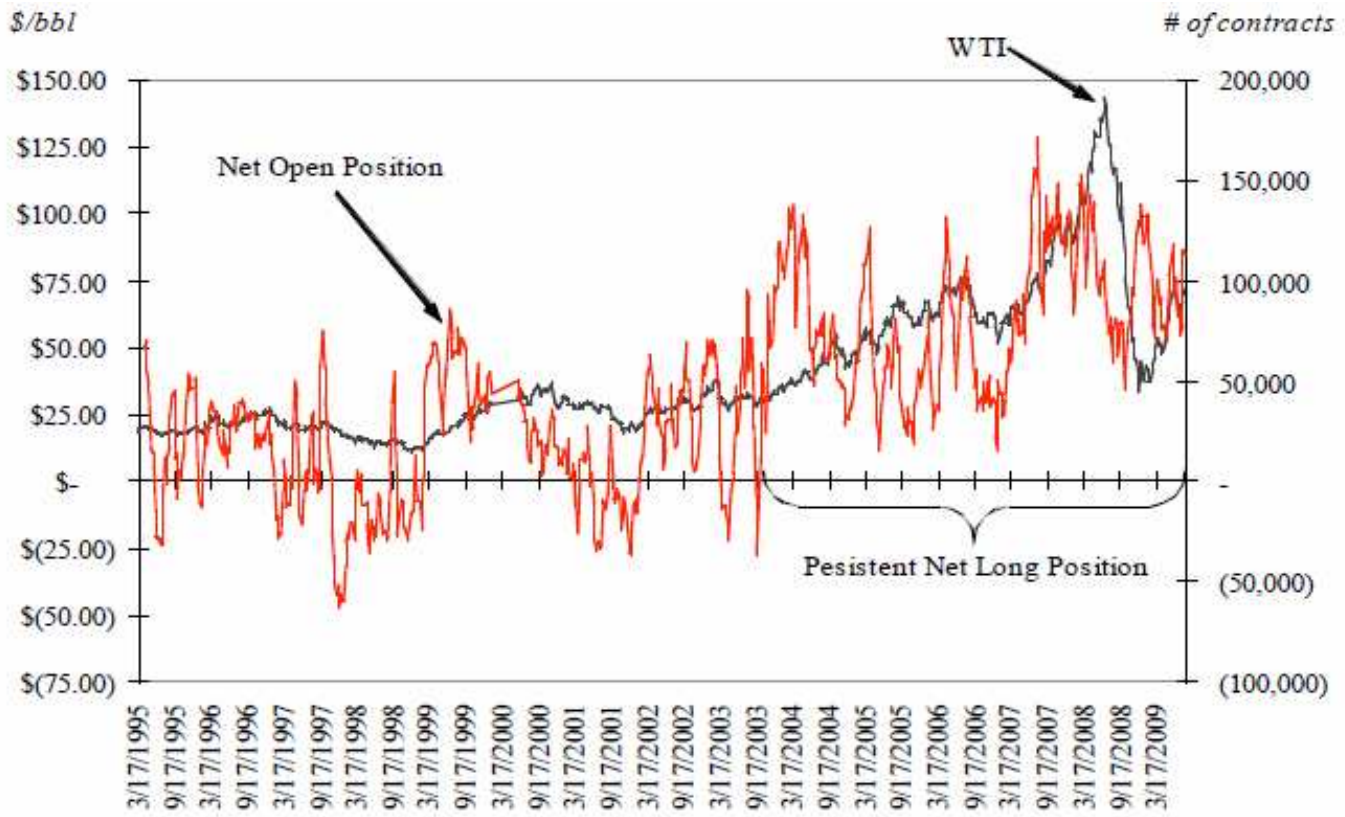

Source: Energy Information Administration, CFTC Commitment of Traders Reports

Source: Medlock et Jaffe (2009)

\section{Déconnexion entre les flux financiers et la réalité physique du marché du pétrole?}

Les échanges de contrats futures sur le NYMEX correspondent à 600 millions de barils, soit sept fois plus que le volume quotidien de demande en pétrole (Medlock et Jaffe (2009)). En 2007, les positions ouvertes sur les contrats futures de pétrole (NYMEX + ICE) correspondaient à environ 5\% de la production totale annuelle de pétrole, $5 \%$ des réserves américaines prouvées, et un dixième de pourcentage des réserves totales prouvées (Parsons, 2009). L'extension de cette analyse à l'épisode de mars-août 2008 est rendue difficile par l'opacité concernant l'état des réserves (leur définition même), et la capacité des agents financiers tels que les «swap dealers» à manipuler les prix des cours de matières premières (voir Parsons (2009) pour plus de détails). 


\section{Qui souffre de la spéculation?}

Hamilton (2009) établit que dans un contexte de hausse des prix de l'essence et de chute du pouvoir d'achat, la crise sur le marché du pétrole a profondément impacté l'économie américaine, avec par exemple 125,000 demandeurs d'emplois supplémentaires enregistrés dans le secteur automobile entre les mois de juillet 2007 et août $2008^{9}$.

Rogoff (2006) établit que les économies en voie de développement souffrent particulièrement des chocs sur le marché du pétrole, tandis que les économies développées telles que les Etats-Unis en souffrent de façon indirecte par le creusement de leur déficit courant qui pose de sérieux problèmes de vulnérabilité de long-terme. L'auteur ajoute que les économies actuelles sont moins sensibles aux chocs sur le marché du pétrole qu'auparavant grâce à une meilleure efficacité énergétique, une plus grande concentration de la consommation de pétrole sur la demande finale, une politique monétaire mieux ancrée, des marchés financiers plus développées (plus profonds), et des marchés du travail plus flexibles. Rogoff (2006) note que la volatilité du prix du pétrole à court et long terme constitue un problème important pour les pays producteurs. Tandis que la volatilité de court terme peut être partiellement neutralisée en utilisant des stock «tampon » de pétrole plus grands, la volatilité de long terme peut être réduite par une plus grande transparence de l'industrie pétrolière et par une plus grande flexibilité des taux de change dans les pays touchés par la volatilité du prix du pétrole.

\section{Épisode de mars-août 2008: bulle spéculative ou non?}

Il convient d'apporter une réponse nuancée à cette question. Caballero et al. (2008) placent la présence d'une «bulle spéculative» sur les marchés des matières premières (pas uniquement pétrole) dans le contexte global de crise financière durant l'été 2008, qui a particulièrement freiné la croissance économique mondiale. Ce ralentissement de l'économie permettrait d'expliquer le retournement de la situation sur les marchés des matières premières, détruisant in fine la possible «bulle » sur ces marchés.

\footnotetext{
9 Hamilton (2009) identifie la crise sur le marché du pétrole comme l'une des variables explicatives de la récession globale de l'économie américaine, parmi les principales explications liées à la bulle immobilière, à la chute de la vente de voitures et plus généralement un comportement de consommation se détériorant.
} 
En référence aux analyses développées précédemment, la présence de nombreux acteurs non-professionnels, donc spéculatifs, donne au marché la liquidité nécessaire pour qu'à chaque instant un producteur/négociant/consommateur puisse trouver la contrepartie nécessaire au bouclage/débouclage d'une opération. La présence spéculative est donc nécessaire pour donner au marché la profondeur nécessaire. Pour considérer l'efficience d'un marché à terme, un ratio physique susceptible d'être arbitré/papier de 5 est un minimum à considérer. Il faut cependant remarquer que l'arrivée massive des agents nonfinanciers sur les marchés futures du pétrole suite aux réformes institutionnelles des années 2000 aux États-Unis a profondément changé la donne, et laisse à penser que la spéculation doit être encadrée.

Les analyses de Büyükşahin et al. (2008) et Parsons (2009) révèlent que de fortes présomptions (taille des positions ouvertes, allongement des maturités des contrats futures, influence prépondérante des agents non-financiers (« swap dealers », hedge funds), lacunes de régulation par la CFTC, manque de transparence des échanges hors places de marché, déconnexion apparente avec la production physique) existent pour caractériser l'épisode de mars-août 2008 de « bulle spéculative ».

Hamilton (2009), lui, conclut que la faible élasticité prix de la demande en essence de l'économie américaine et l'incapacité de la production physique de pétrole à augmenter dans un contexte de forte demande en produits pétroliers constituent deux pistes d'explication plus cohérentes que la spéculation per se. Dans un article antérieur, Hamilton (2008) reconnaissait que parmi les principales explications possibles de cet épisode (spéculation sur les marchés de matières premières, forte demande mondiale, limitations temporelles ou géologiques à l'accroissement de la production, détermination monopolistique du prix par l'OPEP, rôle croissant de la rente de rareté de la ressource pétrole), il faut reconnaître un élément de vérité à chacune d'entre elles, plutôt que de chercher à mettre en avant une explication plutôt qu'une autre. 
Ainsi, (i) la faible élasticité de la demande, (ii) la forte croissance de la demande en provenance de Chine, du Moyen-Orient, et des pays nouvellement industrialisés, et (iii) l'incapacité à accrôitre la production globale seraient les principales causes ${ }^{10}$ derrière la pression à la hausse du prix du pétrole qui ont ensuite favorisé l'émergence d'une spéculation plus large sur les marchés de matières premières.

Dans des analyses économétriques récentes, Kaufmann et Ullman (2009) identifient que la relation de long-terme entre les prix spot et futures du pétrole a changé après septembre 2004. Ils suggèrent que les fondamentaux de marché ont initié une tendance haussière sur le long-terme des prix du pétrole, qui a été exacerbée par les spéculateurs, qui avaient euxmêmes anticipé la probabilité élevée que les prix du pétrole croissent dans le temps. Cifarelli et Paladino (2010), eux, concluent qu'il est difficile de prouver que la spéculation a un effet sur le prix du pétrole, car ceux-ci évoluent également en fonction des fondamentaux propres au marché pétrole. Cependant, les auteurs notent que de fortes hausses/baisses quotidiennes du prix futures du pétrole telles que l'on peut les observer sur le NYMEX ne correspondent pas à des changements de fondamentaux, mais bien à des échanges de matières premières énergétique à but spéculatif.

Notons enfin que la spéculation peut aussi être profitable aux pays producteurs tels que l'Arabie Saoudite: de faibles déclins de production accroissent les revenus courants et entrent dans leur stratégie de long-terme, à mesure que la rente de rareté s'inscrit comme un élément permanent de la détermination du prix du pétrole.

\section{LIMITES DES ANALYSES ACTUELLES}

1. Nous n'avons pas accès aux données non-publiques de la CFTC pour mener une contre-expertise.

2. Nous n'avons pas accès aux volumes échangés sur les marchés options pour étudier la liquidité/l'étendue de la spéculation sur les marchés dérivés pétrole.

3. Aucune analyse académique ne porte sur l'étude des données de volatilité intra-quotidienne durant l'épisode de mars-août 2008, qui correspondent pourtant plus à la réalité des mécanismes de trading.

\footnotetext{
10 C'est aussi la thèse que soutient Krugman (2008) dans sa chronique du New York Times.
} 


\section{Quels instruments de politique économique mettre en æuvre?}

- Le gouvernement américain aurait pu vendre du pétrole à partir de ses réserves stratégiques, au moment où les ventes correspondaient avec l'expiration des contrats futures NYMEX (Hamilton (2009), Medlock et Jaffe (2009)). De façon similaire, les pays développés peuvent s'entendre pour une utilisation coordonnée des réserves stratégiques de pétrole de l'Agence Internationale de l'Energie (AIE).

\section{Encadré 4 : Les politiques mises en œuvre aux États-Unis}

Dans les années 1990, l'administration Clinton a indiqué publiquement aux marchés du pétrole et à l'OPEP qu'elle utiliserait les réserves stratégiques américaines pour limiter le prix du baril de pétrole à $\$ 40$. Cette stratégie a découragé les opérateurs sur les marchés futures à prendre des positions longues au-delà de $\$ 39$.

Une politique inverse a été menée en 2007-2008, conduisant à augmenter les réserves stratégiques de pétrole des États-Unis, ce qui a signifié aux opérateurs de marché et à l'OPEP que les États-Unis n'utiliseraient pas leurs réserves stratégiques de pétrole pour encadrer les fluctuations de prix sur les marchés. Ce comportement a pu nourrir le comportement de spéculation en prenant des positions longues sans crainte de perte en cas d'utilisation surprise des réserves stratégiques de pétrole des États-Unis ou de l'AIE.

- L'utilisation des «spare capacities » des producteurs de pétrole peut également servir à encadrer le prix du pétrole lors d'épisodes de forte volatilité similaires à la période de mars-août 2008 (Medlock et Jaffe (2009)), et lors d'asymétries entre l'offre et la demande de pétrole.

- La re-régulation des marchés futures de pétrole (et notamment les conséquences peut être non intentionnelles du CFMA) apparaît comme un élément central. Il s'agit de repenser le fonctionnement des marchés, et l'influence des fonds d'index de matières premières et des agents «swappant» des matières premières sur ces marchés. L'intervention physique des gouvernements apparaît également nécessaire dans des circonstances extrêmes (Medlock et Jaffe (2009)). Cifarelli et Paladino (2010) concluent leur étude en demandant un renforcement de la restriction sur le comportement de spéculation sur les marchés futures du pétrole par la CFTC. 
- De façon plus conventionnelle, la politique monétaire permet d'encadrer les flambées des prix des matières premières (Hamilton (2009)). Cependant, cet outil de politique économique doit être mobilisé avec une grande prudence. Bernanke, Gertler, Watson (1997) montrent en effet qu'une politique monétaire systématique peut être responsable de la transmission des chocs du marché du pétrole sur l'activité économique, plus que les changements du prix du pétrole per se. L'économie américaine a en effet enregistré un très rapide déclin des taux d'intérêt à court terme durant le premier trimestre 2008. Ce type d'intervention invite à repenser l'action de la Federal Reserve, comme Ben Bernanke (le Chairman) luimême le suggère dans ses déclarations datées de janvier 2009.

- Fattouh et Allsopp (2009) suggèrent l'utilisation d'un autre instrument de politique économique non conventionnel: l'instauration d'une bande de prix du pétrole explicite et basée sur les fondamentaux attendus du marché du pétrole (détaillés précédemment). Cette solution a pour avantage de fournir aux opérateurs de marché une fourchette au sein de laquelle les prix du pétrole fluctuent (ou ont fluctué de façon implicite dans le passé), mais nécessite dans le même temps l'implication des gouvernements dans une « diplomatie du pétrole » difficile à mettre en œuvre.

- Enfin, de façon générale, la littérature académique souligne le besoin d'une plus grande transparence de l'industrie pétrolière concernant l'état des réserves, l'accès aux stocks excédentaires de pétrole, les échanges hors place de marché, et la régulation des agents non-commerciaux sur les marchés dérivés pétrole («swap dealers », hedge funds) (Büyükşahin et al. (2008)).

\section{Conclusion}

La complexité des interactions entre le physique et le financier limite de fait toute explication univoque sur la forte variation des prix du pétrole sur la période récente. Dans l'abondante littérature consacrée au sujet, il est difficile de trancher entre les tenants des fondamentaux physiques (la demande adressée des pays émergents, les craintes d'un «peak oil », la crise économique, etc.) et les tenants des fondamentaux financiers (le rôle des taux de change et des taux d'intérêt, la montée en puissance du pétrole "papier », l'arrivée de nouveaux intervenants tels que les fonds indexés sur les matières premières, les 
comportements moutonniers - herding - des investisseurs, le jeu des arbitragistes spot futures et ses limites).

Les données statistiques disponibles ne permettent pas non plus d'établir clairement des liens de causalité entre les positions ouvertes des investisseurs financiers sur les marchés de futures et les prix observés sur le marché spot. A contrario, rien ne permet également de l'exclure. Ainsi, on peut raisonnablement avancer en conclusion :

- que le jeu de certains acteurs financiers a pu amplifier les mouvements à la hausse ou à la baisse des cours, augmentant la volatilité naturelle des prix du pétrole ;

- qu'on ne peut exclure que de tels mouvements ne se reproduisent dans les années à venir, à la volatilité naturelle s'ajoutant celle due aux comportements d'investisseurs financiers qui considèrent le pétrole (et plus généralement les matières premières) comme une classe d'actifs arbitrables par rapport à d'autres ;

- que les tensions fortes sur les cours se manifesteront en raison essentiellement de fondamentaux physiques (sous-investissement en nouvelles capacités) d'ici la fin de la décennie ;

- que le fonctionnement des marchés financiers du pétrole et la logique financière des acteurs qui les animent sont porteurs de risques difficilement contrôlables qui peuvent engendrer un risque systémique.

La question du prix du pétrole débouche ainsi sur la problématique plus générale de la régulation des marchés financiers. 


\section{Bibliographie}

Andersen, T.G., et Bollerslev, T. 1998. Answering the Skeptics: Yes, Standard Volatility Models do Provide Accurate Forecasts. International Economic Review 39(4), 885-905.

Bank for International Settlements, 2009, Semi-annual OTC Derivatives Statistics At EndJune 2008, Table 19, www.bis.org/statistics/derstats.htm.

Bernanke, B.S., Gertler, M., Watson, M. 1997. Systematic Monetary Policy and the Effects of Oil Price Shocks, Brookings Papers on Economic Activity 1, 91-157.

Blanchard, O.J., Gali, J. 2007. The Macroeconomic Effects of Oil Price Shocks: Why are the 2000s so different from the 1970s? NBER Working Paper \#0517

Blanchard, O.J., Riggi, M. 2009. Why are the 2000s so different from the 1970s? A structural interpretation of changes in the macroeconomic effects of oil prices. NBER Working Paper \#15467

Black, F., et Scholes, M. 1973. The Pricing of Options and Corporate Liabilities, Journal of Political Economy 81, 637-654.

BOE, 2008. What can be said about the rise and fall in oil prices? Bank of England Research and Analysis Report, préparé par Saporta, V., Trott, M., et Tudela, M. 215-225

Bollerslev, T., 1986. Generalized autoregressive conditional heteroskedasticity. Journal of Econometrics 31, 307-327.

Büyükşahin, B., Haigh, M.S., Harris, J.H., Overdahl, J.A., and Robe, M.A. 2008. Fundamentals, Trader Activity and Derivative Pricing. SSRN Working Paper \#966692.

Caballero, R., Farhi, E., Gourinchas, P. 2008, Financial Crash, Commodity Prices and Global Imbalances. Brookings Papers on Economic Activity (2), 1-55. 
Cabinet Office. 2008, The rise and fall in oil prices: analysis of fundamental and financial drivers, UK Cabinet Office, Global Energy Team.

Cifarelli, G., Paladino, G. 2010. Oil price dynamics and speculation: a multivariate financial approach. Energy Economics 32(2), 363-372.

CFTC, 2008, Staff Report on Commodity Swap Dealers and Index Traders with Commission Recommendations, Commodities Futures Trading Commission, Washington D.C., USA.

Cooper, J.C.B. 2003. Price Elasticity of Demand for Crude Oil: Estimates for 23 Countries, OPEC Review 27(1), 1-8.

Dahl, C.A. 1993. A Survey of Oil Demand Elasticities for Developing Countries, OPEC Review 17, 399-419.

Dvir, E., Rogoff, K.S. 2009. The Three Epochs of Oil, Working Paper, Harvard University, USA.

Dumas, B., Kurshev, A. et Uppal, R. 2006. What Can Rational Investors Do About Excessive Volatility and Sentiment Fluctuations? Swiss Finance Institute Research Paper Series \#06 19.

Fattouh, B. et Allsopp, C. 2009. The Price Band and Oil Price Dynamics. Oxford Institute for Energy Studies.

Garman, M., et Klass, M. 1980. On the estimation of security price volatilities from historical data. Journal of Business 53, 67-78.

Hamilton, J.D. 1983. Oil and the Macroeconomy since World War II, Journal of Political Economy 91(2), 228-248.

Hamilton, J.D. 1996. This is what happened to the oil-price macroeconomy relationship, Journal of Monetary Economics 38(2), 215-220. 
Hamilton, J.D. 2008. Understanding Crude Oil Prices. Working Paper, University of California, San Diego, USA.

Hamilton, J.D. 2009. Causes and Consequences of the Oil Shock of 2007-08. Brookings Papers on Economic Activity 1, 215-261.

Kaufmann, R.K., Ullman, B. 2009. Oil prices, speculation, and fundamentals: Interpreting causal relations among spot and futures prices. Energy Economics 31, 550-558.

Kilian, L. 2008a. Exogenous Oil Supply Shocks: How Big Are They and How Much Do They Matter for the U.S. Economy? The Review of Economics and Statistics 90(2), 216240.

Kilian, L. 2008b. A Comparison of the Effects of Exogenous Oil Supply Shocks on Output and Inflation in the G7 Countries, Journal of the European Economic Association 6(1), 78121.

Kilian, L. 2009. Not All Oil Price Shocks Are Alike: Disentangling Demand and Supply Shocks in the Crude Oil Market, The American Economic Review 99(3), 1053-1069.

Kilian, L. 2010. Explaining Fluctuations in Gasoline Prices: A Joint Model of the Global Crude Oil Market and the U.S. Retail Gasoline Market. The Energy Journal 31(2), 105 130.

Krugman, P. 2008. The oil nonbubble, The New York Times, 12 Mai 2008, disponible à l'adresse: www.nytimes.com/2008/05/12/opinion/12krugman.html .

Masters, M.W. 2008. Testimony before the Committee of Homeland Security and Governmental Affairs United States Senate, disponible à l'adresse: www.hsgac.senate.gov/public/_files/052008Masters.pdf.

Masters, M.W. and White, A.K., 2008, The Accidental Hunt Brothers-How Institutional Investors Are Driving Up Food and Energy Prices, at www.accidentalhuntbrothers.com . 
Medlock III, K.B., Jaffe, A.M. 2009. Who is in the oil futures market and how has it changed? Working Paper, Rice University, USA.

Parkinson, M. 1980. The extreme value method for estimating the variance of the rate of return. Journal of Business 53, 61-68.

Parsons, J.E. 2009. Black Gold \& Fool's Gold: Speculation in the Oil Futures Market. MIT CEEPR Working Paper \#09-013.

Rogoff, K.S. 2006. Oil and the Global Economy. Working Paper, Harvard University, USA.

Shiller, R. J., 1981, Do Stock Prices Move Too Much to be Justified by Subsequent Changes in Dividends?, American Economic Review 71, 421-36.

Tang, K., Xiong, W. 2009. Index Investing and the Financialization of Commodities. Working Paper, Princeton University, USA.

Timmermann, A., 1996, Excess Volatility and Predictability of Stock Prices in Autoregressive Dividend Models with Learning, Review of Economic Studies 63, 523-57.

Timmermann, A. G., 1993, How Learning in Financial Markets Generates Excess Volatility and Predictability in Stock Prices, Quarterly Journal of Economics 108, 113545.

U.S. Government Accountability Office, 2007. Trends in Energy Derivatives Markets Raise Questions about CFTC Oversight. U.S. Government Accountability Office, GAO 0825, disponible à l'adresse suivante : http://www.gao.gov/products/GAO-08-174T

Zivot, E., and Andrews, D.W.K. 1992. Further evidence on the Great Crash, the Oil-Price Shock, and the Unit-Root Hypothesis. Journal of Business \& Economic Statistics 10(3), 251-270. 